\title{
Avaliação do Processo de Secagem de Uva cv. Arra 15®
}

\author{
Evaluation of the Drying Process of Grape cv. Arra 15®
}

Evaluación del Proceso de Secado de la Uva cv. Arra 15®

Recebido: 14/04/2021 | Revisado: 21/04/2021 | Aceito: 22/04/2021 | Publicado: 08/05/2021

Silvana Belém de Oliveira Vilar

ORCID: https://orcid.org/0000-0001-8141-3373 Instituto Federal de Educação, Ciência e Tecnologia do Sertão Pernambucano, Brasil

E-mail: silvana.belem@ifsertao-pe.edu.br

Nailton de Macedo Albuquerque Junior

ORCID: https://orcid.org/0000-0001-6757-5114

Universidade Federal de Campina Grande, Brasil E-mail: junior.nailton99@gmail.com

Aurianna Barros Coelho

ORCID: https://orcid.org/0000-0001-6747-9560 Instituto Federal de Educação, Ciência e Tecnologia do Sertão Pernambucano, Brasil

E-mail: aurianna.coelho@ifsertao-pe.edu.br

Joselane Nascimento da Silva

ORCID: https://orcid.org/0000-0002-9338-4423

Instituto Federal de Educação, Ciência e Tecnologia do Sertão Pernambucano, Brasil E-mail: joselanens13@hotmail.com

Sâmela Leal Barros

ORCID: https://orcid.org/0000-0003-2047-4636 Universidade Federal do Ceará, Brasil E-mail: samelaleal7@gmail.com

Wilton Pereira da Silva

ORCID: https://orcid.org/0000-0001-5841-6023

Universidade Federal de Campina Grande, Brasil

E-mail:wiltonps@uol.com.br

\section{Resumo}

O Brasil é um país no qual possui uma grande diversidade de frutas, com um destaque no cultivo de uva, principalmente na região nordeste, porém devido a elevada exportação da fruta, boa parte considerada imprópria é descartada. Com isso o presente estudo objetivou elaborar uva passa da $\mathrm{cv}$. Arra $15^{\prime}$ ( ) a partir da secagem em estufa e air fryer com a aplicação de um pré-tratamento químico, e caracterizar quanto as propriedades físico-químicas. As uvas in natura foram pré-tratadas com $\mathrm{NaOH} 1 \%$ a $95^{\circ} \mathrm{C}$ durante 30 segundos, secas em estufa e air fryer. A fruta foi caracterizada quanto à umidade, sólidos solúveis totais, $\mathrm{pH}$, acidez total e cor. Os processos de secagem utilizados reduziram a umidade, obtendo assim a uva passa. O modelo de logaritmo se ajustou melhor aos dados experimentais. Com o aumento da temperatura houve o aumento da difusividade, e a partir da solução analítica da equação de difusão apresentou valores baixos de número de Biot, indicando que a condição de contorno utilizada (terceiro tipo) não descreveu de forma satisfatória o processo. Com isso conclui-se que estes resultados são importantes pois demostram que o processamento foi eficiente e possibilitou a obtenção da uva passa com boas características nutricionais.

Palavras-chave: Caracterização; Novas cultivares; Pré-tratamento; Uva passa.

\begin{abstract}
The Brazil is a country where there is a great diversity of fruits, with an emphasis on grape growing, especially in the northeast region, but due to the high export of fruit, much of it considered improper is discarded. Therefore, this study aimed to elaborate raisin grape of $c v$. Arra $15^{\prime}($ from the drying in oven and air fryer with the application of a chemical pre-treatment, and to characterize how much the physical-chemical properties. The grapes in natura were pre-treated with $\mathrm{NaOH} 1 \%$ to $95{ }^{\circ} \mathrm{C}$ for 30 seconds, dried in oven and air fryer. The fruit was characterized as to humidity, total soluble solids, $\mathrm{pH}$, total acidity and color. The drying processes used reduced the humidity, thus obtaining the raisin. The logarithm model was better adjusted to the experimental data. With the increase in temperature there was an increase in diffusivity, and from the analytical solution of the diffusion equation presented low values of Biot number, indicating that the boundary condition used (third type) did not describe the process satisfactorily. With this, we conclude that these results are important because they demonstrate that the processing was efficient and made it possible to obtain the raisin with good nutritional characteristics.
\end{abstract}

Keywords: Characterization; New cultivars; Pre-treatment; Raisin. 


\section{Resumen}

Brasil es un país en el que tiene una gran diversidad de frutas, con énfasis en el cultivo de la uva, especialmente en la región nordeste, pero debido a la alta exportación de la fruta, se descarta gran parte de la que se considera inadecuada. Así, el presente estudio tuvo como objetivo la elaboración de pasas del $c v$. Arra 15'® a partir del secado en horno y freidora de aire con la aplicación de un pretratamiento químico, y la caracterización de las propiedades fisicoquímicas. Las uvas frescas se pretrataron con $\mathrm{NaOH}$ al $1 \%$ a $95{ }^{\circ} \mathrm{C}$ durante 30 segundos, se secaron en horno y en freidora. La fruta se caracterizó en cuanto a humedad, sólidos solubles totales, $\mathrm{pH}$, acidez total y color. Los procesos de secado utilizados redujeron la humedad, obteniendo así la pasa. El modelo logarítmico es el que mejor se ajusta a los datos experimentales. Con el aumento de la temperatura se produjo un incremento de la difusividad, y a partir de la solución analítica de la ecuación de difusión se presentaron valores bajos del número de Biot, indicando que la condición de contorno utilizada (tercer tipo) no describía satisfactoriamente el proceso. Así, se concluye que estos resultados son importantes porque demuestran que el procesamiento fue eficiente y permitió obtener pasas con buenas características nutricionales.

Palabras clave: Caracterización; Nuevos cultivares; Pretratamiento; Pasas.

\section{Introdução}

O Brasil tem notoriedade na produção de frutas, a qual aumenta a cada ano, por ser um país que possui uma elevada biodiversidade, sendo favorecida pela sua localização geográfica (Abrafrutas, 2017). Uma das frutas em destaque no país é a uva, a qual compõe a cadeia produtiva nacional (Debastiani et al., 2015). No entanto, o cultivo de uva no Vale do São Francisco tem como principal foco a exportação, cujos padrões de qualidade são estabelecidos pelo país de destino, o que acarreta uma elevada quantidade de uvas "de descarte", por não atenderem aos padrões de qualidade estabelecidos para a exportação (Lima, 2012).

A região do Submédio do Vale do São Francisco possui características que favorecem a produção da uva como o clima, solo adequado e baixa umidade. A disponibilidade e utilização de um sistema de irrigação, favorece o cultivo e proporciona maior produtividade de frutos com ótima qualidade durante o ano todo, devido a obtenção de mais de uma safra anual. Estes fatores deram a região do Vale do São Francisco uma posição de destaque no cenário de produção nacional e internacional (Santos et al., 2011; Silva et al., 2015).

Diante esses atributos que favorecem o cultivo frutífero do Vale do São Francisco, novas variedades estão sendo introduzidas, como a cv. Arra 15' ${ }^{\circledR}$. De um modo geral, a produção tem intuito de encontrar um método de plantio adequado as condições locais, bem como novas formas de aumentar as características, melhorar a qualidade das uvas para o consumo in natura e determinar o ponto de colheita adequado para a comercialização, tendo em vista que é uma variedade que apresenta propriedades como grandes bagas, sem sementes e atraentes, com uma coloração de casca verde-clara e brilhante, em cachos de tamanho médio a grande (Karniel \& Giumarra, 2011).

A uva é apontada como uma matéria-prima de elevada importância, tendo em vista que apresenta elevada qualidade nutricional. A presença de compostos antioxidantes evidência que a incorporação de uvas na dieta proporciona diversos benefícios à saúde humana, além do consumo da fruta in natura, a uva pode ser processada para se obter diversos produtos alimentícios (Lins \& Sartori, 2014; Vilas Boas et al., 2016).

A industrialização de frutos tem como objetivo principal realizar o desenvolvimento de produtos com maior valor agregado e maior vida de prateleira. Através da secagem torna-se possível a elaboração de frutas desidratadas, que são produtos com elevada aceitabilidade. Devido às suas vantagens nutricionais, as passas são consumidas em grandes quantidades no Brasil e podem proporcionar saúde e bem-estar, além de serem utilizadas como ingredientes adicionais em outros alimentos, sendo incorporadas em alimentos como cereais, bolos, sorvetes e iogurtes (Souza et al., 2015; Jeszka-Skowron et al., 2017).

A elaboração de uva passa é uma alternativa viável para que haja a diminuição das perdas que ocorrem na pós-colheita, intensificando também a comercialização regional. Para a elaboração de uva passa, a matéria-prima precisa ser submetida ao processo de secagem ou desidratação, que é um método de conservação que diminui a quantidade de água da fruta ou do vegetal, 
reduzindo a chance de deterioração microbiana, reações enzimáticas e oxidação, sem perdas das características organolépticas do produto (Feiden et al., 2015).

Diante a importância do desenvolvimento de novos produtos e da conservação das suas propriedades, este trabalho objetivou obter uma uva passa cv. ARRA $15^{\circledR}$ através do processo de secagem em estufa e Air fryer, aplicando um prétratamento químico, e avaliar as propriedade físico-química do produto in natura e desidratado.

\section{Metodologia}

O presente trabalho foi desenvolvido no Instituto Federal de Educação, Ciência e Tecnologia do Sertão Pernambucano, Campus Petrolina-PE, no Laboratório Experimental de Alimentos (LEA), Laboratório de Química Analítica e Laboratório de Águas e Bebidas. O tratamento metodológico foi caracterizado de forma quantitativa, onde fez-se a coleta de dados através de medições de grandezas, gerando dados que podem ser analisados por meio de artifícios matemáticos como porcentagens, estatísticas e probabilidades, além de métodos numéricos, métodos analíticos e geração de equações e/ou fórmulas matemáticas aplicáveis a algum processo (Pereira et al., 2018).

\subsection{Matéria-prima}

Nesse estudo utilizou-se como matéria-prima a uva $c v$. Arra $15^{\prime} \circledR$ doadas pela Fazenda Esperança, localizada no Projeto Senador Nilo Coelho, Núcleo 4, Petrolina-PE. Os frutos maduros e considerados como de "descarte" pela fazenda foram imediatamente transportados para o Laboratório Experimental de Alimentos (LEA) do IF Sertão-PE. Ao chegarem ao LEA foram selecionados e higienizados com cloro à 200 ppm por 15 min, enxaguados com água potável e secos com toalhas absorventes para retirada do excesso de água. Após sanitização foram retiradas amostras da uva para a caracterização in natura das matériaprima.

\subsection{Pré-tratamento químico (Pelagem cáustica)}

Para realizar o pré-tratamento químico foi pesado 2000 gramas da cv. Arra $15^{`}$ ( , que foram mergulhadas em solução de hidróxido de sódio $(\mathrm{NaOH} 1 \%)$, à $95^{\circ} \mathrm{C}$ durante 30 segundos, posteriormente as bagas foram enxaguadas em água corrente para retirar o excesso da solução alcalina, quando a água de lavagem ficou transparente na presença de fenolftaleína finalizouse a lavagem das frutas. Retirou-se o excesso da água com toalhas absorventes.

\subsection{Cinética de Secagem}

Após o pré-tratamento químico foram pesadas 1000 gramas de uva para o processo de secagem no secador de bandejas com circulação de ar forçada modelo LE 30 a $65{ }^{\circ} \mathrm{C} \pm 5$, e 1000 gramas para a fritadeira elétrica Cadence® (modelo FRT531127/FRT531-220) a $90^{\circ} \mathrm{C}$, as pesagens das amostras para os dois métodos de secagem foram realizadas em balança semi-analítica a cada 15 minutos nas três primeiras horas e posteriormente a cada 30 minutos até atingir o peso constante.

As curvas de secagem foram obtidas pela conversão dos dados referentes à perda de água ao parâmetro adimensional razão do conteúdo de água (RX) de acordo com a Equação 1.

$$
R X=\frac{X-X_{e}}{X_{0}-X_{e}}
$$


Onde,

RX - razão de teor de água (adimensional);

$\mathrm{X}$ - teor de água, base seca;

Xe - teor de água de equilíbrio, base seca;

X0 - teor de água inicial, base seca.

Os dados experimentais das cinéticas de secagem foram ajustados aos modelos matemáticos dispostos na Tabela 1, usando-se o programa computacional Statistica ${ }^{\circledR}$ versão 7.0 através de regressão não linear.

Tabela 1. Modelos matemáticos utilizados para estimar as curvas de cinética de secagem da $\mathrm{cv}$. Arra $15^{\text {' }}$ (

\begin{tabular}{ccc}
\hline Designação & Modelo \\
\hline Exponencial Dois Termos & $\mathrm{RX}=\mathrm{a} \cdot \exp (-\mathrm{kt})+(+\mathrm{a}) \exp (-\mathrm{kat})$ \\
Henderson \& Pabis & $\mathrm{RX}=\mathrm{a} \exp (-\mathrm{kt})$ \\
Logarítmico & $\mathrm{RX}=\mathrm{a} \cdot \exp (-\mathrm{kt})+\mathrm{c}$ \\
Midilli & $\mathrm{RX}=\mathrm{a} \cdot \exp \left(-\mathrm{kt}^{\mathrm{n}}\right)+\mathrm{bt}$ \\
Page & $\mathrm{RX}=\exp \left(-\mathrm{kt}^{\mathrm{n}}\right)$
\end{tabular}

RX - Razão de teor de água, adimensional; a, b, c, k, n - constantes dos modelos; $\mathrm{t}$ - tempo de secagem (min). Fonte: Autores (2021).

Foram utilizados como critérios de ajuste dos modelos matemáticos aos dados experimentais a magnitude do coeficiente de determinação (R2) (Equação 2) e desvio quadrático médio (DQM) (Equação 3).

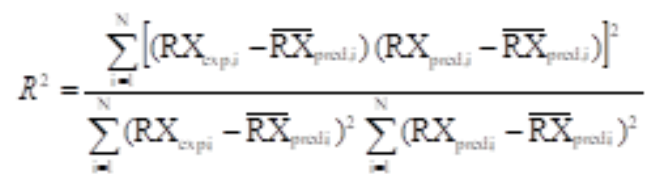

Sendo,

R2 - coeficiente de determinação;

RXexp - razão de teor de água experimental;

$\overline{\mathrm{RX}}_{\text {pred,i }}$ - média da razão de teor de água experimental;

RXpred - razão de teor de água predita pelo modelo;

$\overline{\mathrm{RX}}_{\text {pred,i }}$ - média da razão de teor de água predita pelo modelo;

$\mathrm{N}$ - número de observações.

$$
\mathrm{DQM}=\left[\frac{1}{\mathrm{~N}} \sum_{i=1}^{\mathrm{N}}\left(\mathrm{RX}_{\mathrm{predi}}-\mathrm{RX}_{\operatorname{expi}}\right)^{2}\right]^{\frac{1}{2}}
$$

Onde,

DQM - desvio quadrático médio; 
RXpred - razão de teor de água predita pelo modelo;

RXexp - razão de teor de água experimental;

$\mathrm{N}$ - número de observações.

De posse dos dados de teor de água (b.s.) da $c v$. Arra $15^{\prime}($. em cada tempo de secagem, foi calculada as taxas de secagem, de acordo com a Equação 4.

$$
\mathrm{TX}=\frac{\mathrm{X}_{\mathrm{t}_{0}}-\mathrm{X}_{\mathrm{t}_{0}+\Delta t}}{\Delta \mathrm{t}}
$$

Sendo,

TX - taxa de secagem kg kg-1.min-1;

Xto - teor de água no tempo anterior, $\mathrm{kg} \mathrm{kg-1} \mathrm{(b.s.);}$

$\mathrm{Xto}+\Delta \mathrm{t}$ - teor de água no tempo atual, $\mathrm{kg} \mathrm{kg-1} \mathrm{(b.s.);}$

$\Delta \mathrm{t}$ - diferença entre o tempo atual (ti) e o anterior (t0) de secagem, min.

\subsubsection{Taxa de secagem}

As taxas de secagem foram obtidas através do cálculo da derivada do modelo de Page, conforme expresso a seguir na Equação 5:

$$
\frac{d R x}{d t}=-K n t^{n-1} \exp \left(-k t^{n}\right)
$$

O software LabFit Ajuste de Curvas foi utilizado para obtenção dos gráficos (Silva \& Silva, 2009a).

\subsubsection{Equação de difusão}

A equação de difusão para a secagem das uvas, utilizando a geometria de cilindro infinito pode ser escrita como:

$$
\frac{\partial R X}{\partial t}=\frac{1}{r} \frac{\partial}{\partial r}\left(r D \frac{\partial R X}{\partial r}\right)
$$

Em que D é a difusividade efetiva de massa e r é a posição no cilindro infinito referente ao ponto axial.

As simulações foram realizadas com relação as cinéticas de secagem das uvas utilizando o software "Convective Adsorption-Desorption 2.5" (Silva \& Silva, 2009b), que fornece uma solução analítica e utiliza a condição de contorno do terceiro tipo.

\subsection{Caracterização Físico-química}

As uvas $c v$. Arra $15^{\prime} 囚$. foram caracterizadas na sua forma in natura e desidratada em triplicata quanto aos seguintes parâmetros físico-químicos: teor de umidade que foi determinado pelo método de secagem das amostras em estufa a $105^{\circ} \mathrm{C}$ até peso constante, sólidos solúveis totais (SST) foi determinado a partir da leitura do índice de refração no refratômetro ABBE, pH determinado em pHmetro e acidez total titulável (ATT) por titulometria seguindo os procedimentos propostos pelo Instituto Adolfo Lutz (Brasil, 2008) e as coordenadas cromáticas L* que representa a luminosidade, transição do branco (0) para o preto (100), a** que representa a transição da cor verde $\left(-a^{*}\right)$ para a cor vermelha $\left(+a^{*}\right)$ e b** a transição da cor azul (-b*) para a cor 
amarela $\left(+b^{*}\right)$ foram determinadas por leitura utilizando-se colorímetro HunterLab XE Plus, modelo 4500 L, com sistema de cor Cielab.

\subsection{Análise estatística}

Os dados obtidos com relação a caracterização físico-química das amostras foram avaliados estatisticamente, pela análise descritiva, teste shapiro-wilk para normalidade, e as medias comparadas pelo teste de Tukey a 5\% de probabilidade, com o auxílio do programa estatístico ASSISTAT versão 7.7beta (Silva \& Azevedo, 2016).

\section{Resultados e Discussões}

Através da Tabela 1, observa-se os parâmetros de ajuste aos dados experimentais obtidos através dos modelos empíricos de Page, Handerson e Pabis, Midili, Exponencial de dois termos e Logaritmo.

Tabela 1. Parâmetros de ajuste obtidos através dos modelos de Page, Handerson e Pabis, Midili, Exponencial de dois termos e Logaritmo, com seus respectivos coeficientes de determinação (R2) e desvio quadrático médio (DQM) da secagem de uvas cv. Arra $15^{\prime}{ }^{\circledR}$. em secador de bandeja e Air fryer.

\begin{tabular}{|c|c|c|c|c|c|c|c|}
\hline \multirow{2}{*}{ Experimentos } & \multicolumn{7}{|c|}{ Parâmetros para o modelo de Page } \\
\hline & - & - & $\mathrm{k}$ & - & $\mathrm{n}$ & $\mathrm{R}^{2}$ & DQM \\
\hline Air Fryer-SPQ & - & - & 0,0013 & - & 1,2845 & 0,9994 & $1,19 \times 10^{-2}$ \\
\hline Air Fryer-CPQ & - & - & 0,0014 & - & 1,3261 & 0,9975 & $2,25 \times 10^{-2}$ \\
\hline Secador de bandeja-SPQ & - & - & 0,0004 & - & 1,1359 & 0,9914 & $2,92 \times 10^{-2}$ \\
\hline Secador de bandeja-CPQ & - & - & 0,0008 & - & 1,1001 & 0,9944 & $2,01 \times 10^{-1}$ \\
\hline \multirow{2}{*}{ Experimentos } & \multicolumn{7}{|c|}{ Parâmetros para o modelo de Henderson \& Pabis } \\
\hline & $\mathrm{a}$ & - & $\mathrm{k}$ & - & - & $\mathrm{R}^{2}$ & DQM \\
\hline Air Fryer-SPQ & 1,0796 & - & 0,0065 & - & - & 0,9942 & $4,33 \times 10^{-2}$ \\
\hline Air Fryer-CPQ & 1,0811 & - & 0,0074 & - & - & 0,9902 & $5,02 \times 10^{-2}$ \\
\hline Secador de bandeja-SPQ & 1,0811 & - & 0,0012 & - & - & 0,9878 & $7,42 \times 10^{-2}$ \\
\hline Secador de bandeja-CPQ & 1,0385 & - & 0,0018 & - & - & 0,9957 & $4,94 \times 10^{-2}$ \\
\hline \multirow{2}{*}{ Experimentos } & \multicolumn{7}{|c|}{ Parâmetros para o modelo de Midili } \\
\hline & $\mathrm{a}$ & $\mathrm{b}$ & $\mathrm{k}$ & - & $\mathrm{n}$ & $\mathrm{R}^{2}$ & DQM \\
\hline Air Fryer-SPQ & 1,0991 & 0,0004 & 0,0149 & - & 0,7969 & 0,9948 & $1,93 \times 10^{-2}$ \\
\hline Air Fryer-CPQ & 1,0145 & 0,0001 & 0,0250 & - & 1,1829 & 0,9981 & $2,29 \times 10^{-4}$ \\
\hline Secador de bandeja-SPQ & 1,0524 & 0,0001 & 0,0021 & - & 0,8310 & 0,9985 & $2,38 \times 10^{-2}$ \\
\hline Secador de bandeja-CPQ & 1,0690 & 0,0001 & 0,0061 & - & 0,7695 & 0,9977 & $4,44 \times 10^{-2}$ \\
\hline \multirow{2}{*}{ Experimentos } & \multicolumn{7}{|c|}{ Parâmetros para o modelo Exponencial de dois termos } \\
\hline & $\mathrm{a}$ & - & $\mathrm{k}$ & - & - & $\mathrm{R}^{2}$ & DQM \\
\hline Air Fryer-SPQ & 0,0054 & - & 1,0991 & - & - & 0,9896 & $1,32 \times 10^{-2}$ \\
\hline Air Fryer-CPQ & 0,0058 & - & 1,1351 & - & - & 0,9858 & $2,71 \times 10^{-2}$ \\
\hline Secador de bandeja-SPQ & 0,0024 & - & 0,2843 & - & - & 0,9820 & $2,14 \times 10^{-2}$ \\
\hline Secador de bandeja-CPQ & 0,0059 & - & 0,4520 & - & - & 0,9942 & $3,83 \times 10^{-2}$ \\
\hline \multirow{2}{*}{ Experimentos } & \multicolumn{7}{|c|}{ Parâmetros para o modelo Logaritmo } \\
\hline & $\mathrm{a}$ & - & $\mathrm{k}$ & $\mathrm{c}$ & - & $\mathrm{R}^{2}$ & DQM \\
\hline Air Fryer-SPQ & 1,1729 & - & 0,0050 & 0,1278 & - & 0,9988 & $2,41 \times 10^{-5}$ \\
\hline Air Fryer-CPQ & 1,2060 & - & 0,0053 & 0,1641 & - & 0,9970 & $1,03 \times 10^{-6}$ \\
\hline Secador de bandeja-SPQ & 1,4630 & - & 0,0006 & 0,4329 & - & 0,9994 & $7,44 \times 10^{-3}$ \\
\hline Secador de bandeja-CPQ & 1,1446 & - & 0,0013 & 0,1376 & - & 0,9995 & $1,93 \times 10^{-3}$ \\
\hline
\end{tabular}

Nota: CPQ- Com pré-tratamento químico/com pelagem; SPQ: Com pré-tratamento químico/ sem pelagem; R2- coeficiente de determinação; DQM- desvio quadrático médio. Fonte: Autores (2021). 
Observou-se que todos os modelos matemáticos apresentam coeficientes de determinação (R2) iguais ou superiores a 0,98 nas temperaturas do ar de secagem aplicadas, entretanto os modelos de Page e Logaritmo obtiveram valores superiores de $\mathrm{R}^{2}$ (0,9914-0,9995). De acordo com Moscon et al. (2017), o coeficiente de determinação (R2), o qual varia de 0 a 1, indica a proximidade entre os dados obtidos e os estimados, verificando-se que todos os modelos aplicados representam adequadamente aos processos de secagem estudados.

No entanto, segundo Martins et al. (2015) o $\mathrm{R}^{2}$ quando avaliado de maneira isolada, não constitui um bom critério de seleção de modelos não-lineares. Ao avaliar o erro quadrático médio (DQM), verificou-se que os menores valores foram para o modelo de Logaritmo quando comparados aos outros modelos, sendo considerado como o mais eficiente para descrever o processo de secagem da uva cv. Arra $15^{\prime}{ }^{\circledR}$. No entanto, deve-se ressaltar que os modelos de Page, Henderson e Pabis, Midili e Exponencial de Dois Termos, também apresentaram altos valores de $\mathrm{R}^{2}$ e baixos valores de DQM, indicando adequação para representar a cinética de secagem da uva cv. Arra 15’®.

Quanto ao parâmetro "k" para cada modelo aplicado observou que com o aumento da temperatura o seu valor também aumentou, devido que uma temperatura mais alta leva a uma taxa de secagem maior, onde o teor de umidade de equilíbrio é alcançado em um menor tempo de contato do produto ao ar de secagem. Observa-se que o parâmetro "a" das equações empíricas de Hedenrson e Pabis, Exponencial de dois Termos e Logaritmo, sofre tendência de aumento com a elevação da temperatura do ar na secagem por Air fryer, e para a secagem em bandejas este parâmetro tende a aumentar nos modelos de Midili e Exponencial de dois Termos.

Nas Figuras de 1 a 5, estão representadas as curvas obtidas dos modelos matemáticos aplicados (Logaritimico, Page, Handerson e Pabis, Midili e Exponencial de dois termos) ajustados aos dados experimentais da cinética de secagem da uva cv. Arra $15^{\prime}{ }^{\circledR}$. nas temperaturas de $70^{\circ} \mathrm{C}$ (secador de bandejas) e $90^{\circ} \mathrm{C}$ (Air fryer).

Figura 1. Ajuste do modelo Logaritmo aos dados da secagem de uvas com e sem pré-tratamento químico em secador de bandeja $\left(70{ }^{\circ} \mathrm{C}\right)$ e em Air fryer $\left(90^{\circ} \mathrm{C}\right)$.

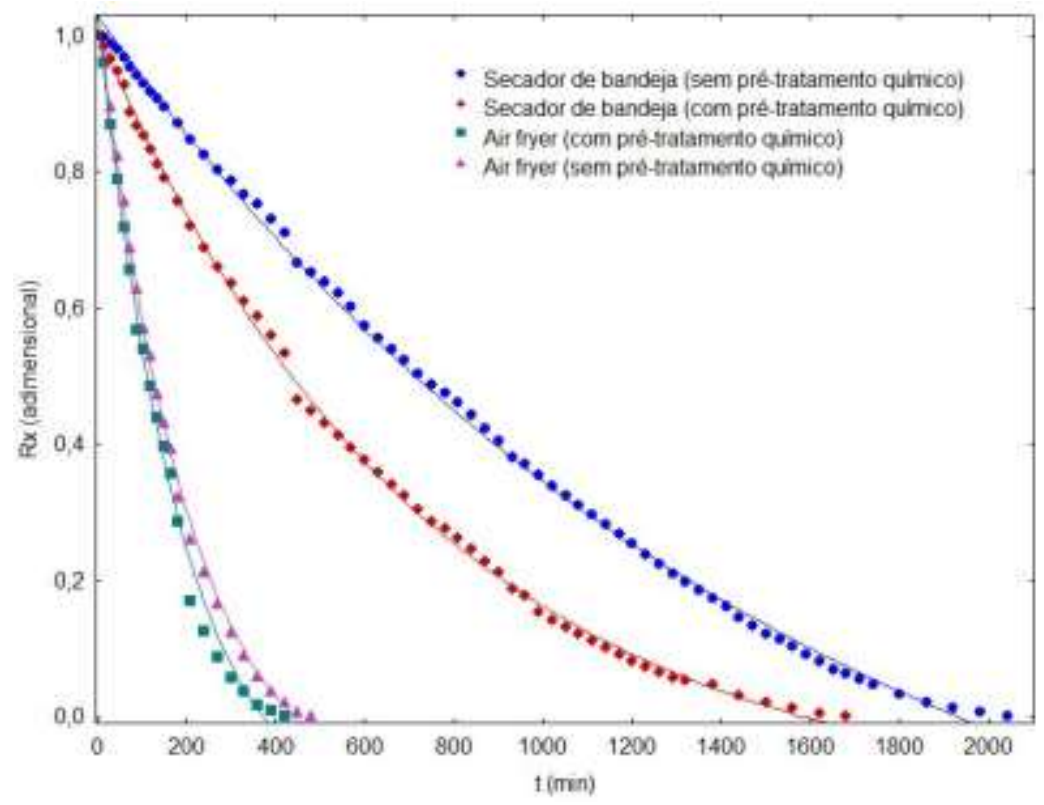

Fonte: Autores (2021). 
Figura 2. Ajuste do modelo de Page aos dados da secagem de uvas com e sem pré-tratamento químico em secador de bandeja $\left(70{ }^{\circ} \mathrm{C}\right)$ e em Air fryer $\left(90^{\circ} \mathrm{C}\right)$.

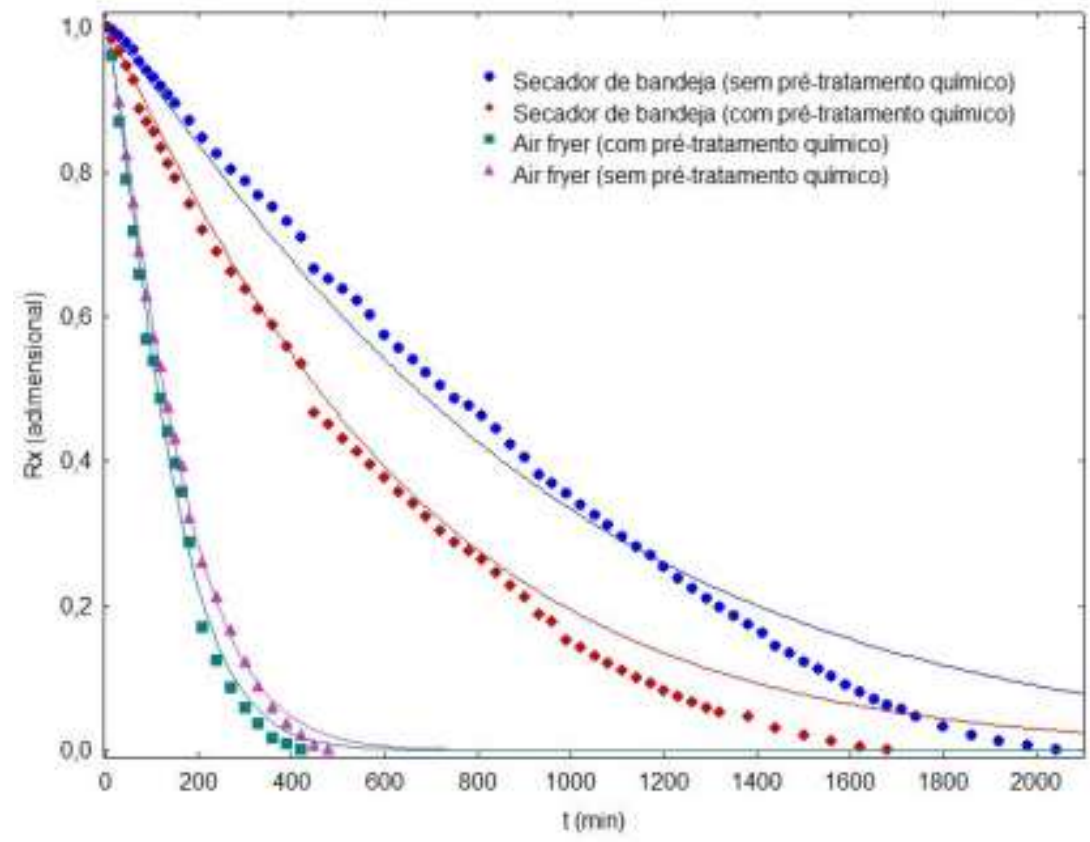

Fonte: Autores (2021).

Figura 3. Ajuste do modelo de Henderson \& Pabis aos dados da secagem de uvas com e sem pré-tratamento químico em secador de bandeja $\left(70^{\circ} \mathrm{C}\right)$ e em Air fryer $\left(90^{\circ} \mathrm{C}\right)$.

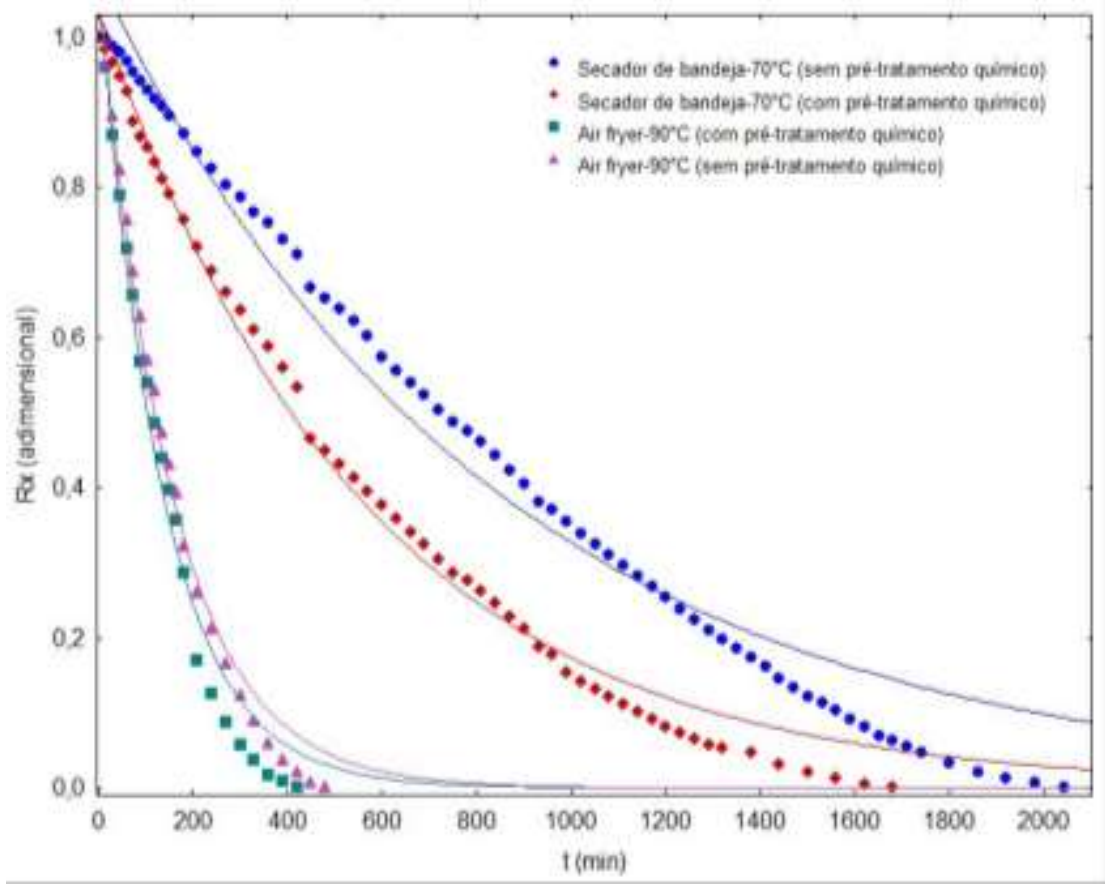

Fonte: Autores (2021). 
Figura 4. Ajuste do modelo de Midili aos dados da secagem de uvas com e sem pré-tratamento químico em secador de bandeja $\left(70{ }^{\circ} \mathrm{C}\right)$ e em Air fryer $\left(90^{\circ} \mathrm{C}\right)$.

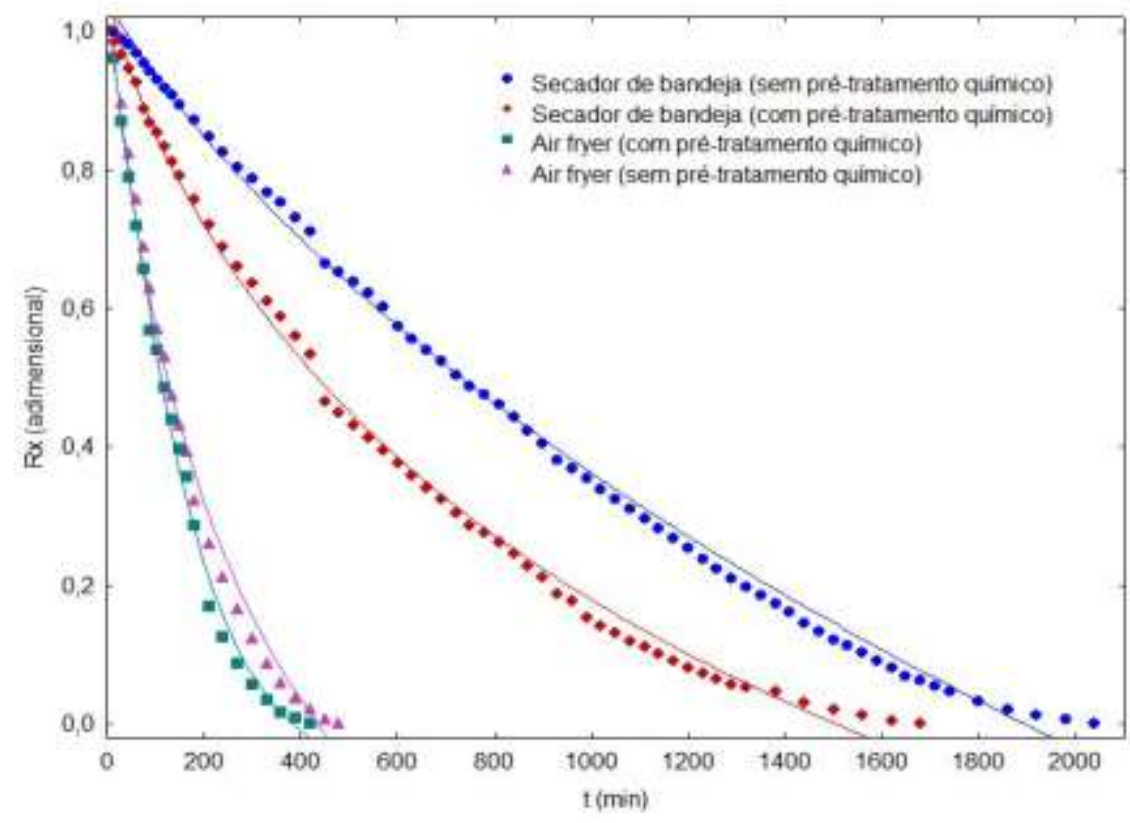

Fonte: Autores (2021).

Figura 5. Ajuste do modelo Exponencial de dois termos aos dados da secagem de uvas com e sem pré-tratamento químico em secador de bandeja $\left(70^{\circ} \mathrm{C}\right)$ e em Air fryer $\left(90^{\circ} \mathrm{C}\right)$.

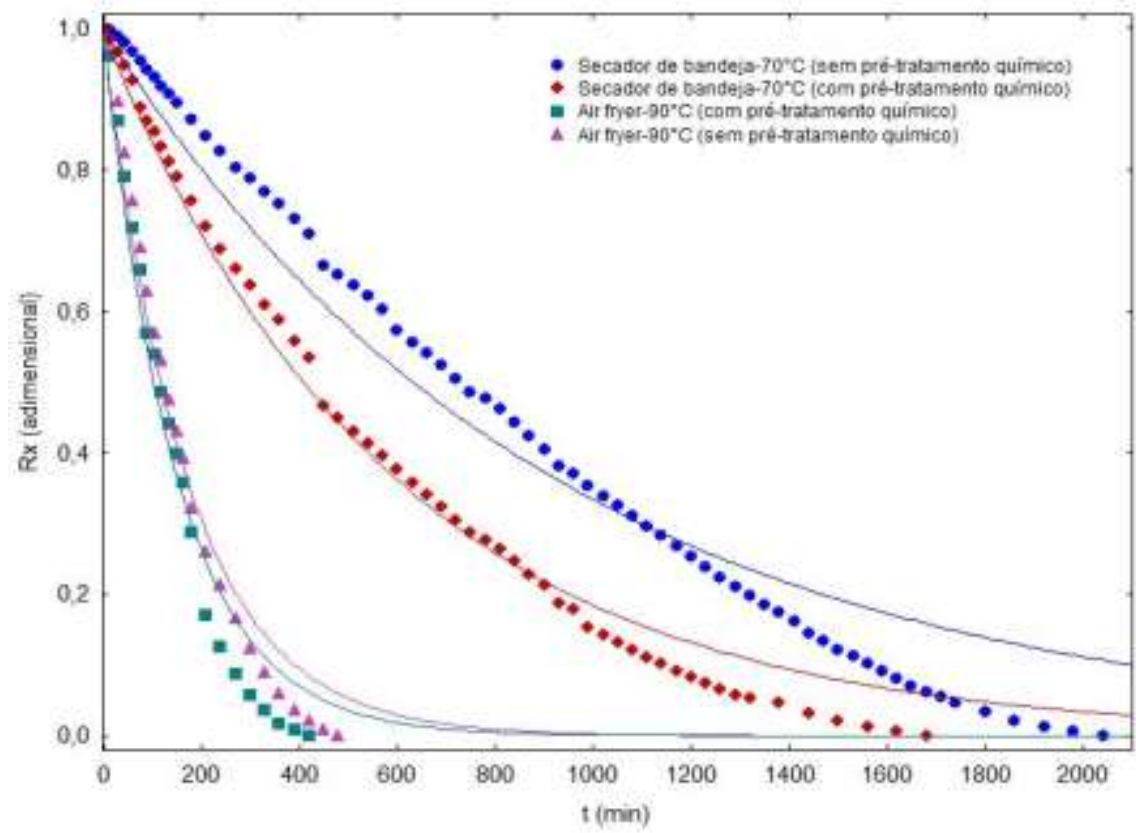

Fonte: Autores (2021).

Após obter os dados experimentais a partir da secagem e calculado a razão de umidade ao longo do tempo nas temperaturas utilizadas, foram ajustados quanto aos modelos matemáticos. A temperatura do ar de secagem usado no processo teve influência nas curvas de secagem, pois quanto maior a temperatura mais rápido se obtém a umidade de equilíbrio constante, fato corroborado por Kaveh et al. (2017), devido ao fato de que a umidade da superfície evapora muito rapidamente devido aos 
altos coeficientes de transferência de calor e massa nos processos de secagem em camada fina, salientando que a taxa de secagem reduz exponencialmente quando toda a umidade da superfície evapora e a secagem se difunde para dentro do material.

Verifica-se nas figuras acima que os modelos testados em ambos os processos de secagem resultaram em bons ajustes aos dados experimentais, com uma melhor representação do fenômeno de secagem da uva cv. Arra $15^{\prime}$ ', devido a curva ajustada pelos modelos terem se aproximado dos resultados experimentais, o que confirma o desempenho deles.

Na Figura 6, está representada as taxas de secagem obtidas do modelo matemático de Page aplicados nas temperaturas de $70{ }^{\circ} \mathrm{C}$ (Secador de bandejas) e $90^{\circ} \mathrm{C}$ (Air fryer).

Figura 6. Taxas de secagem obtida através da derivada do modelo Page para a secagem das uvas em Air fryer e secador de bandeja, com e sem pré-tratamento químico.

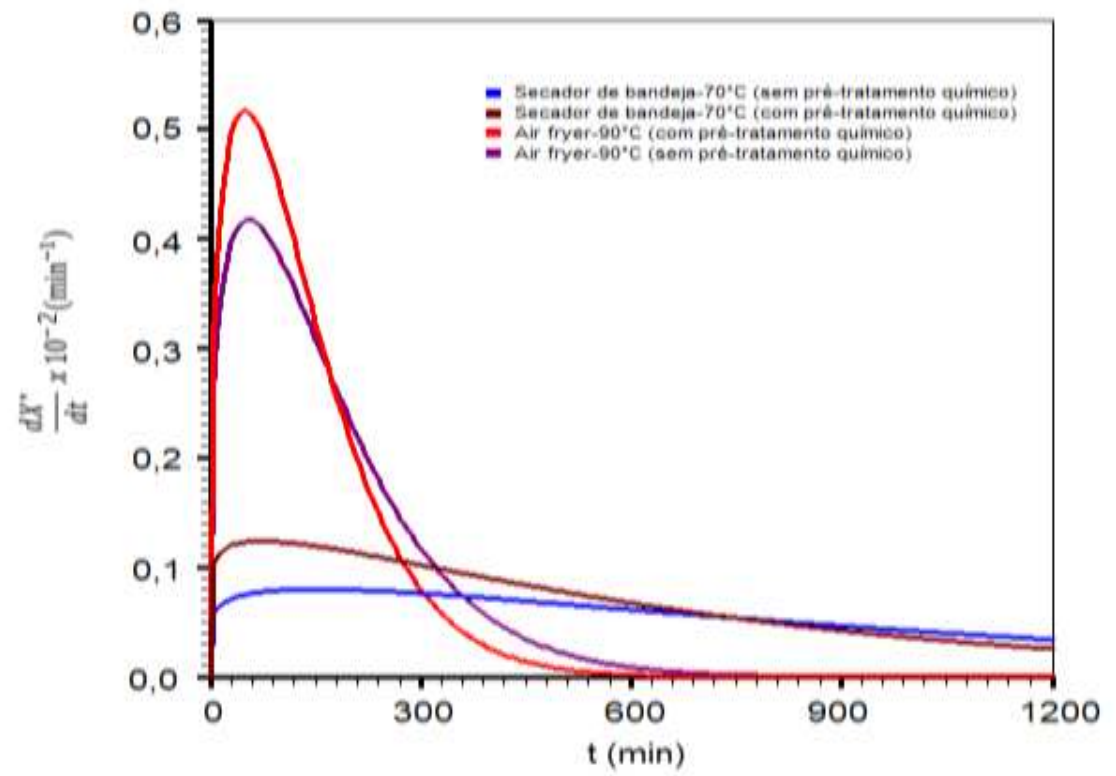

Fonte: Autores (2021).

O resultado obtido observado na figura acima revelou que quanto mais alta a temperatura, mais curto é o tempo de secagem. Os valores obtidos para as taxas de secagem mudam com o tempo, aumentando gradualmente até atingir o valor máximo, sendo seguido por uma brusca diminuição. Isso devido que no início da secagem, a difusão do líquido é o principal mecanismo de transporte da água e, à medida que a secagem avança, a difusão do vapor passa a ser o principal meio, aumentando assim a velocidade de secagem. Porém, com a continuidade do processo, a amostra torna-se insaturada e a difusão da umidade diminui, portanto, a taxa de secagem também diminui (Chen et al., 2017).

Tabela 2. Parâmetros obtidos através do modelo de difusão utilizando solução analítica e condição de contorno do terceiro tipo.

\begin{tabular}{cccccc}
\hline Experimento & $\mathbf{D}\left(\mathbf{m}^{\mathbf{2}} \cdot \mathbf{m i n}^{-\mathbf{1}}\right)$ & $\mathbf{h}\left(\mathbf{m} \cdot \mathbf{m} \mathbf{n i n}^{-\mathbf{1}}\right)$ & $\boldsymbol{B i}$ & $\mathbf{R}^{\mathbf{2}}$ & $\chi^{2}$ \\
\hline Air Fryer-SPQ & $7,07 \times 10^{-6}$ & $1,94 \times 10^{-5}$ & $1,75 \times 10^{-2}$ & 0,9949 & 0,0421 \\
Air Fryer-CPQ & $8,03 \times 10^{-6}$ & $2,21 \times 10^{-5}$ & $1,75 \times 10^{-2}$ & 0,9891 & 0,0571 \\
Secador de bandeja-SPQ & $1,29 \times 10^{-6}$ & $3,57 \times 10^{-6}$ & $1,75 \times 10^{-2}$ & 0,9831 & 0,0229 \\
Secador de bandeja-CPQ & $2,03 \times 10^{-6}$ & $5,58 \times 10^{-6}$ & $1,75 \times 10^{-2}$ & 0,9994 & 0,0531 \\
\hline
\end{tabular}

Nota: CPQ- Com pré-tratamento químico/com pelagem; SPQ: Com pré-tratamento químico/sem pelagem; R2- coeficiente de determinação; $\chi^{2}$-qui-quadrado; D- difusividade efetiva; h- coeficiente convectivo de transferência de calor.

Fonte: Autores (2021). 
A Tabela 2 acima apresenta os coeficientes de difusão efetivos (Deff) e os coeficientes de transferência de massa convectiva (h) obtidos para a secagem das amostras submetidas às temperaturas de $70{ }^{\circ} \mathrm{Ce} 90^{\circ} \mathrm{C}$ com e sem pelagem. Com o aumento da temperatura de secagem convectiva $\left(70-90^{\circ} \mathrm{C}\right)$, há o incremento nos valores de Deff. Além disso, observou-se que amostras submetidas às soluções com a mesma temperatura, mas com o pré-tratamento químico, em particular para a temperatura de secagem de $90^{\circ} \mathrm{C}$, oferecem maior resistência à transferência externa de massa, o que por sua vez pode estar relacionado à redução do coeficiente convectivo de transferência de calor (h).

Observa-se que o número de Biot (Bi) apresentou-se na faixa de 0,001 a 12,500 (Tabela 2), o que segundo Kaya et al. (2010), é indicativo da existência de resistências internas e externas à transferência de água, sendo considerado o caso mais realista em aplicações práticas. Ressalta-se que o número de Biot (Bi) permaneceu igual em ambas temperaturas e processo de secagem com a elevação da temperatura de secagem, indicando que há uma mesma e maior resistência ao escoamento de massa na superfície das amostras (Silva et al., 2013).

Os valores obtidos a partir da triplicata das análises centesimais das uvas in natura, antes do processo de secagem e prétratamento com $\mathrm{NaOH}$, estão destacados na Tabela 3.

Tabela 3. Caracterização físico-química da uva $c$. Arra $15^{\text {'}}$ in natura.

\begin{tabular}{cc}
\hline ANÁLISE & RESULTADOS \\
\hline Umidade (\%) & $79,27 \pm 0,02$ \\
\hline $\mathrm{pH}$ & $3,5 \pm 0,01$ \\
\hline${ }^{\circ}$ Brix & $19,3 \pm 0,36$ \\
\hline Acidez Titulável & $7,9 \pm 0,04$ \\
\hline \multirow{2}{*}{ Cor } & $43,6 \pm 1,34$ \\
& $-5,01 \pm 0,15$ \\
\hline
\end{tabular}

Fonte: Autores (2021).

O teor de umidade obtido para a uva in natura foi de $79,27 \%$, este valor é aceitável por se tratar de uma fruta com alto teor de umidade, mas deve-se observar que os teores de umidade dos frutos são influenciados por fatores como região de cultivo, características do solo, clima, época de colheita e grau de maturação do fruto, que também podem interferir nas características físicas e químicas da fruta (Morzelle et al., 2015).

A uva in natura apresentou $\mathrm{pH}$ correspondente a 3,5, desta forma a uva se caracteriza como um alimento ácido. De acordo com Vilar et al. (2020), o pH dos alimentos é um atributo que depende de vários fatores, entre eles, o estado de preservação e condições das matérias-primas utilizadas e um pH ácido é ótimo do ponto de vista microbiológico.

O teor de sólidos solúveis é um dos indicadores de qualidade mais utilizados (Sun et al., 2017). De acordo com Brasil (2018) uvas que tem como destino o processamento, necessita-se ter no mínimo $14^{\circ}$ Brix, com isso a Arra $15^{`}{ }^{\circledR}$ utilizada encontra-se acima do valor estabelecido, estando adequada para o processo.

A cor é um importante atributo de qualidade de frutas frescas e processadas, pois a cor está intimamente relacionada à aceitação dos alimentos pelo consumidor, sendo o aspecto visual o principal fator decisivo na escolha de um produto (Ribeiro et al., 2012).

Os valores obtidos a partir da triplicata das análises centesimais das uvas desidratadas, após os processos de secagem estão destacados na Tabela 04. 


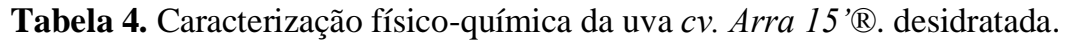

\begin{tabular}{|c|c|c|c|c|c|c|}
\hline \multirow{3}{*}{ Experimento } & \multicolumn{6}{|c|}{ Resultados } \\
\hline & \multirow{2}{*}{$\mathrm{pH}$} & \multirow{2}{*}{${ }^{\circ}$ Brix } & \multirow{2}{*}{ AT } & \multicolumn{3}{|c|}{ Cor } \\
\hline & & & & $\mathrm{L}^{*}$ & $a^{*}$ & $b^{*}$ \\
\hline $\begin{array}{c}\text { Air Fryer } \\
\text { (CP) }\end{array}$ & $3,7 \mathrm{a} \pm 0,0$ & $66 \mathrm{a} \pm 0,0$ & $2,7 \mathrm{a} \pm 0,0$ & $17,9 b \pm 0,0$ & $8,7 b \pm 0,0$ & $8,7 b \pm 0,0$ \\
\hline \multirow[t]{2}{*}{$\begin{array}{c}\text { Air Fryer } \\
\quad(\mathrm{SP})\end{array}$} & $3,7 \mathrm{a} \pm 0,0$ & $63,7 b \pm 0,6$ & $2,8 \mathrm{a} \pm 0,0$ & $22,6 a \pm 0,4$ & $10,6 \mathrm{a} \pm 0,0$ & $11,9 b \pm 0,0$ \\
\hline & \multicolumn{6}{|c|}{ Resultados } \\
\hline \multirow[t]{2}{*}{ Experimento } & \multirow{2}{*}{$\mathrm{pH}$} & \multirow{2}{*}{${ }^{\circ}$ Brix } & \multirow{2}{*}{$\mathrm{AT}$} & \multicolumn{3}{|c|}{ Cor } \\
\hline & & & & $\mathrm{L}^{*}$ & $\mathrm{a}^{*}$ & $b^{*}$ \\
\hline \multicolumn{7}{|l|}{ Secador de } \\
\hline $\begin{array}{c}\text { Bandejas } \\
\text { (CP) }\end{array}$ & $3,9 \mathrm{a} \pm 0,0$ & $63,3 \mathrm{a} \pm 1,2$ & $2,2 \mathrm{a} \pm 0,0$ & $19,3 b \pm 0,1$ & $7,8 \mathrm{a} \pm 0,0$ & $11,1 \mathrm{a} \pm 0,0$ \\
\hline \multicolumn{7}{|l|}{ Secador de } \\
\hline $\begin{array}{c}\text { Bandejas } \\
\text { (SP) }\end{array}$ & $3,9 \mathrm{a} \pm 0,0$ & $62,3 a \pm 1,2$ & $2,0 \mathrm{a} \pm 0,0$ & $22,6 a \pm 0,0$ & $7,2 \mathrm{a} \pm 0,0$ & $10,9 \mathrm{a} \pm 0,0$ \\
\hline
\end{tabular}

Fonte: Autores (2021).

Sabe-se que o potencial hidrogeniônico é um dos parâmetros mais importantes para avaliação de alimentos, pois indica se os produtos são seguros em relação ao crescimento de microrganismos (Vilar et al. 2020). Comparando o processo de secagem pelo Air fryer e pré-tratamento pode-se observar que os valores de $\mathrm{pH}$ não apresentaram diferença significativa entre elas, mostrando valores médios confiáveis $(3,9)$ por apresentarem desvio padrão reduzidos.

Assim como o pH, os sólidos solúveis é um atributo importante na avaliação de alimentos, uma vez que esse parâmetro apresenta a quantidade de açúcares e outros sólidos solúveis presentes na amostra (Gouveia et al., 2014).

Observou-se que o pré-tratamento com $\mathrm{NaOH}$ influenciou significativamente no teor de sólidos solúveis da secagem convectiva, que variou de $66^{\circ}$ Brix (para a amostra com pré-tratamento) a 63, $7^{\circ}$ Brix (para a amostra sem pré-tratamento), tendo em vista que a desidratação da uva concentrou mais esses compostos. No caso das uvas desidratadas no air fryer não foram observadas diferenças estatísticas significativas com relação a este parâmetro, que variou de 62,3 a $63,3{ }^{\circ}$ Brix.

Quanto a acidez titulável, Carranza-Concha et al. (2012), identificou que geralmente a acidez de uvas submetidas a secagem após pré-tratamento com $\mathrm{NaOH}$ apresentaram perdas significativas, o que foi explicado pela diminuição de ácidos, como o ácido ascórbico, que, de acordo com os autores, podem ter sido causadas pela lixiviação durante o tempo de imersão das bagas e degradação do ácido em meio alcalino, fato observado no presente estudo sendo que no secador de bandejas não houve diferença significativa, e no air fryer os resultados diferiram estatisticamente. Em relação as coordenadas de cor houve a redução no experimento com pelagem influenciando na intensificação do escurecimento da uva passa.

Em relação a secagem pelo secador de bandejas com e sem pelagem, pode-se observar que os parâmetros pH, Acidez titulável e ${ }^{\circ}$ Brix não se diferiram estatisticamente entre eles, apresentando resultados seguros devido aos valores baixos de desvio padrão. Quanto as coordenadas de cor a* e b* também não houve diferença significativa entre os tratamentos, já para os valores de Luminosidade foi observado diferença significativa mostrando a mudança de cor no produto após a secagem. Segundo Rocha 
e Reed (2014), a cor é determinada pela presença de pigmentos e alguns deles podem desempenhar um papel importante na saúde, diante disso mesmo após o processo de secagem em ambos os tratamentos as uvas-passas apresentaram valores significativos, principalmente, para as que foram secas no secador de bandejas devido a temperatura ter sido inferior ao da utilizada no Air fryer, acometendo em menos perdas nos parâmetros analisados.

\section{Conclusão}

O processo de secagem reduziu o teor de umidade e a atividade de água da uva $c v$. Arra $15^{\prime} \circledast$, possibilitando a obtenção da uva passa, assim como a aplicação de pré-tratamento químico não provocou mudanças significativas na maioria dos parâmetros de qualidade avaliados em uvas, garantindo assim uma vida útil mais longa e um produto de maior valor agregado.

Os modelos matemáticos aplicados apresentaram bons ajustes em relação aos dados experimentais obtidos, entretanto o modelo de Logaritmo apresentou ajuste superior em comparação aos outros modelos testados, por apresentar valores superiores do R2 e valores inferiores de DQM.

A solução analítica de cilindro infinito apresentou aumento da difusividade e do coeficiente convectivo de transferência de calor com o aumento da temperatura, e baixos valores do número de Biot que indicam que a condição de contorno utilizada (terceiro tipo) não descreveu de forma satisfatória o processo, tendo em vista que o número de Biot foi muito menor que 0,1 .

Por meio de análises físico-químicas de uvas in natura $c v$. Arra $15^{\prime} \circledast$, conclui-se que a fruta possui características adequadas para ser utilizada na fabricação de uvas passas, além disso, o uso do refugo pode reduzir o descarte e, assim, diminuir o desperdício, obtendo um produto de maior valor agregado.

Aconselha-se a utilização de outros pré-tratamentos, bem como o uso de outras técnicas de secagem, uma vez que a uva cv. Arra $15^{\prime}$ é uma variedade nova na região do Submédio do Vale do São Francisco e apresenta boas propriedades para a elaboração de novos subprodutos.

\section{Agradecimentos}

Ao Instituto Federal do Sertão Pernambucano (IF-Sertão), pela concessão da bolsa de iniciação científica.

\section{Referências}

ABRAFRUTAS - Associação Brasileira dos Produtores Exportadores de Frutas e Derivados (2017). Dados estatísticos do setor: Estatísticas das exportações de frutas no $1^{\circ}$ semestre de 2017; Agronegócio exportou U\$ 45 bilhões no primeiro semestre, alta de 4\%. <http://abrafrutas.org>.

Carranza-Concha, J., Benlloch, M., Camacho, M. M., \& Martíneznavarrete, N. (2012). Effects of Drying and Pretreatment on the Nutritional and Functional Quality of Raisins. Food and Bioproducts Processing, 90(2), 243-248.

Chen, Q., Bi, J., Chen, R., Liu, X., Wu, X., \& Zhou, M. (2017). Estudo comparativo sobre as características de secagem, difusividade da umidade e alguns atributos físicos e nutricionais de fatias de cenoura escaldadas. Journal of Food Process and Preservation, 41, 1-8.

Debastiani, G., Leite, A. C., Weiber Junior, C. A., \& Boelhouwer, D. I. (2015). Cultura da Uva, Produção e Comercialização de Vinhos no Brasil: Origem, Realidades e Desafios. Revista Cesumar Ciências Humanas e Sociais Aplicadas, 20(2), 471-485.

Feiden, A., Feiden, A., Galvani, F., \& Campolin, A. (2015). Desidratação de frutas utilizando secador solar: Embrapa Pantanal. 5 p. (Comunicado Técnico 98).

Gouveia, A. M. S., Correia, C. V., Tavares, A. E., Evangelista, R. M., \& Cardoso, A. I. I. (2014). Qualidade de Raízes de Batata-Doce em Função da Adubação Nitrogenada e Conservação. Revista Raízes e Amidos Tropicais, 10(1), 57- 64.

Jeszka-Skowron, M., Zgola-Grześkowiak, A., Stanisz, E., \& Waśkiewicz, A. (2017). Potential health benefits and quality of dried fruits: goji fruits, cranberries and raisins. Food Chemistry, 221, 228-236.

Karniel, E. S., \& Giumarra, E. S. (2011). Grape Plant Named “Arrafifteen”. US Pat. 2011/0219502 P1, 8 set. 4 p.

Kaveh, M., Chayjan, R. A., \& Nikbakht, A. M. (2017). Mass transfer characteristics of eggplant slices during length of continuous band dryer. Heat and Mas s Transfer, 53(6), 2045-2059.

Kaya, A., Aydín, O., \& Dincer, I. (2010). Comparação de dados experimentais com resultados de alguns modelos de secagem para produtos de forma regular. Heat Mass Transfer, 46, 555-562. 
Lima, M. S. (2012). Estudo dos atributos sensoriais de uva passa submetida a tratamentos para manutenção da cor pós-secagem. VII CONEPPI- Congresso Norte Nordeste de pesquisa e inovação, Palmas- TO, out.

Lins, A. R., \& Sartoni, G. V. (2014). Qualidade Fenólica e Atividade Antioxidante de Vinhos Tintos Produzidos no Estado do Paraná. Revista Brasileira de Produtos Agroindustriais, 16(1), 69-76.

Lorenzi, C. O., Julião, L., Santos, F. G. R., \& Oliveira, F. V. (2014). Embalagens das “de sempre” para as sofisticadas. Hortifruti Brasil. 12(137), 08-23.

Martins, E. A. S., Lage, E. Z., Goneli, A. L. D., Hartmann Filho, C. P., \& Lopes, J. G. (2015). Cinética de secagem de folhas de timbó (Serjania marginata Casar). Revista Brasileira de Engenharia Agrícola e Ambiental, 19(3), 238-244. 10.1590/1807-1929/agriambi. v19n3p238-244.

Morzelle, M. C., Bachiega, P., Souza, E. C., Vilas Boas, E. V. B., \& Lamounier, M. L. (2015). Caracterização química e física de frutos de curriola, gabiroba e murici provenientes do cerrado brasileiro. Revista Brasileira de Fruticultura, 37, 96- 103.

Moscon, E. S., Martin, S., Spehar, C. R., Devilla, I. A., \& Rodolfo Junior, R. (2017). Cinética de secagem de grãos de quinoa (chenopodium quinoa w.). Revista Engenharia na Agricultura, 25(4), 318-325.

Pereira, A. S., Shitsuka, D. M., Parreira, F. J., \& Shitsuka, R. (2018). Metodologia da Pesquisa Cientifica. (1 ${ }^{\mathrm{a}}$ ed.): UFSM, NTE.

Ribeiro, T. P., Lima, M. A. C., \& Alves, R. E. (2012). Maturação e qualidade de uvas para suco em condições tropicais, nos primeiros ciclos de produção. Pesquisa Agropecuaria brasileira, Brasília, 47(8), 1057-1065.

Rocha, D. S, \& Reed, E. (2014). Pigmentos Naturais em Alimentos e sua Importância para a Saúde. Estudos Vida e Saúde, 41(1), 76-85.

Robertson, G. L. (2009). Food packaging and shelf life: a practical guide: CRC Press.

Santos, E. H. B., Azevêdo, L. C., Batista, F. P. R., Matos, L. P., \& Lima, M. S. (2011). Caracterização química e sensorial de uvas desidratadas, produzidas no Vale do São Francisco para infusão. Revista Semiárido De Visu, 1(2), 134-147.

Silva, A. C. G. C., Fontes, C. H. O., \& Barbosa, A. S. (2015). Multicriteria evaluation model for organizational performance management applied to the Polo Fruit Exporter of the São Francisco Valley. Computers and Electronics in Agriculture, 117, 168-176.

Silva, G. S., Santos, S. P. S., Barbosa, N. F. P., Santos, R. G., Bery, C. S., \& Silva, G. F. (2015). Secagem e caracterização físico-química da uva Crimson. Universidade Federal de São Carlos. XXXVII ENEMP, Out.

Vilar, S. B. O., Silva, J. N., Oliveira, I. P., Albuquerque, N. M. J., \& Coelho, A. B. (2020). Isoterma de Sorção de uva arra 15® desidratada e armazenada em diferentes embalagens. Congresso internacional da Agroindústria.

Silva, W. P., \& Silva, C. M. D. P. S. (2009). “Convective Adsorption-Desorption 2.5” software. Disponível no endereço eletrônico http://zeus.df.ufcg.edu.br/labfit/Convective.htm

Silva, W. P., Silva, \& C. M. D. P. S. (2009) LAB Fit Curve Fitting Software, V.7.2.46. www.labfit.net, data de acesso: 05 de novembro de 2018.

Silva, W. P., Silva, C. M. D. P. S., \& Gomes, J. P. (2013). Descrição da secagem de pedaços cilíndricos de banana em diferentes temperaturas usando modelos de difusão. Journal of Food Engineering, 117, 417-424.

Souza, R. T., Cornejo, F. E. P., Nogueira, R. I., Freitas, D. G. C., Protas, J. F. S., Maia, J. D. G., Mattos, C. T. G. B., Leal Junior, W. F., \& Ritschel, P. S. (2015). Uvas-passas Brasileiras: Matéria-prima e Processamento. Circular Técnica 115. Embrapa, ISSN 1516-5914, Bento Gonçalves, RS.

Sun, M., Zhang, D., Liu, L., \& Wang, Z. (2017). How to predict the sugariness and hardness of melons: A near-infrared hyperspectral imaging method. Food Chemistry, 218, 413-421.

Zenebon, O., Pascuct, N. S., \& Tiglea, P. (2008). Métodos físico-químicos para análise de alimentos. (4ª Ed.): Instituto Adolfo Lutz. 